\title{
A New Design for Measuring Potentials in Operando Nanoelectronic Devices by Electron Holography
}

\author{
Kai $\mathrm{He}^{1,2}$ and John Cumings ${ }^{1}$ \\ 1. Department of Materials Science and Engineering, University of Maryland, College Park, MD 20742 \\ 2. Center for Functional Nanomaterials, Brookhaven National Laboratory, Upton, NY 11973
}

Electron holography $(\mathrm{EH})$ based on transmission electron microscope (TEM) is widely recognized as a powerful interferometric technique to image and quantify electric and magnetic fields for a variety of physical and chemical applications [1]. Off-axis EH has been successfully applied for accurate quantitative two-dimensional (2D) mapping of intrinsic electrostatic potentials arising from material-dependent mean inner potentials or semiconductor's dopant profiles, where the target objects are in the stationary state and isolated from external electric bias [2]. But for measuring exotic properties of an electrically-biased material or an operando device, a global electric field has to be inevitably involved, which is typically nonuniform and also nonlinear to applied voltages. In these scenarios, reference background approximation was often used but sometimes tended to cause nonnegligible artifacts. Thus, it is critical to develop a new strategy to overcome these drawbacks and achieve more accurate measurements.

In this study, we propose a new design of in-situ electric biasing platform for precise nanoscale potential measurement of operando nanoelectronic devices using EH technique [3]. In this design, we use a pair of parallel metal lines as sourcing electrodes. Their lengths are sufficiently longer than the spacing width, so as to generate uniform electric field transversely along the electrodes (analogous to a planar capacitor) and provide theoretically identical phase background in both object region and reference region. The design was implemented into a prototype biasing platform on a customized silicon nitride TEM membrane with help of lithography, etching and deposition, as shown in Fig. 1a. We utilized a multiwalled carbon nanotube (MWCNT) as a resistive testing device and recorded holograms on the CNT device in operando mode (Fig. 1b). The projected 2D phase maps can be extracted for different external biases and matched with calculations (Fig. 1c), which exhibit a linear dependence between phase gradient and applied voltage (Fig. 1d). From the distribution of the phase shifts, we can quantify the potential profile along the CNT. With additional finite element modelling, we can further determine the detailed transport properties, such as separating potential drop attributed to the CNT itself and that due to the contact resistances.

More interestingly, it is noted that the asymmetric feature of the EH phase shift can be used as a simple signature for quick and non-volatile diagnosis of uneven resistivity caused by localized contact failures. This approach can also be generally implemented to a broad range of novel nanomaterials and nanodevices for measuring their intrinsic transport properties as well as understanding the overall functionality. In summary, the methodology and scientific justification demonstrated in this study offer a promising pathway for exploring fundamental science by electron holography [4].

\section{References:}

[1] M R McCartney and D J Smith, Annu. Rev. Mater. Res. 37 (2007) 729.

[2] M R McCartney et al, Ultramicroscopy 110 (2001) 375.

[3] K He and J Cumings, Nano Lett. 13 (2013) 4815. 
[4] The authors acknowledge funding support from U.S. Department of Energy under Award Number DE-FG02-10ER46742 and Award Number DESC0001160, as well as use of facilities in Maryland NanoCenter and its NispLab supported in part by NSF MRSEC Grant DMR 05-20471.
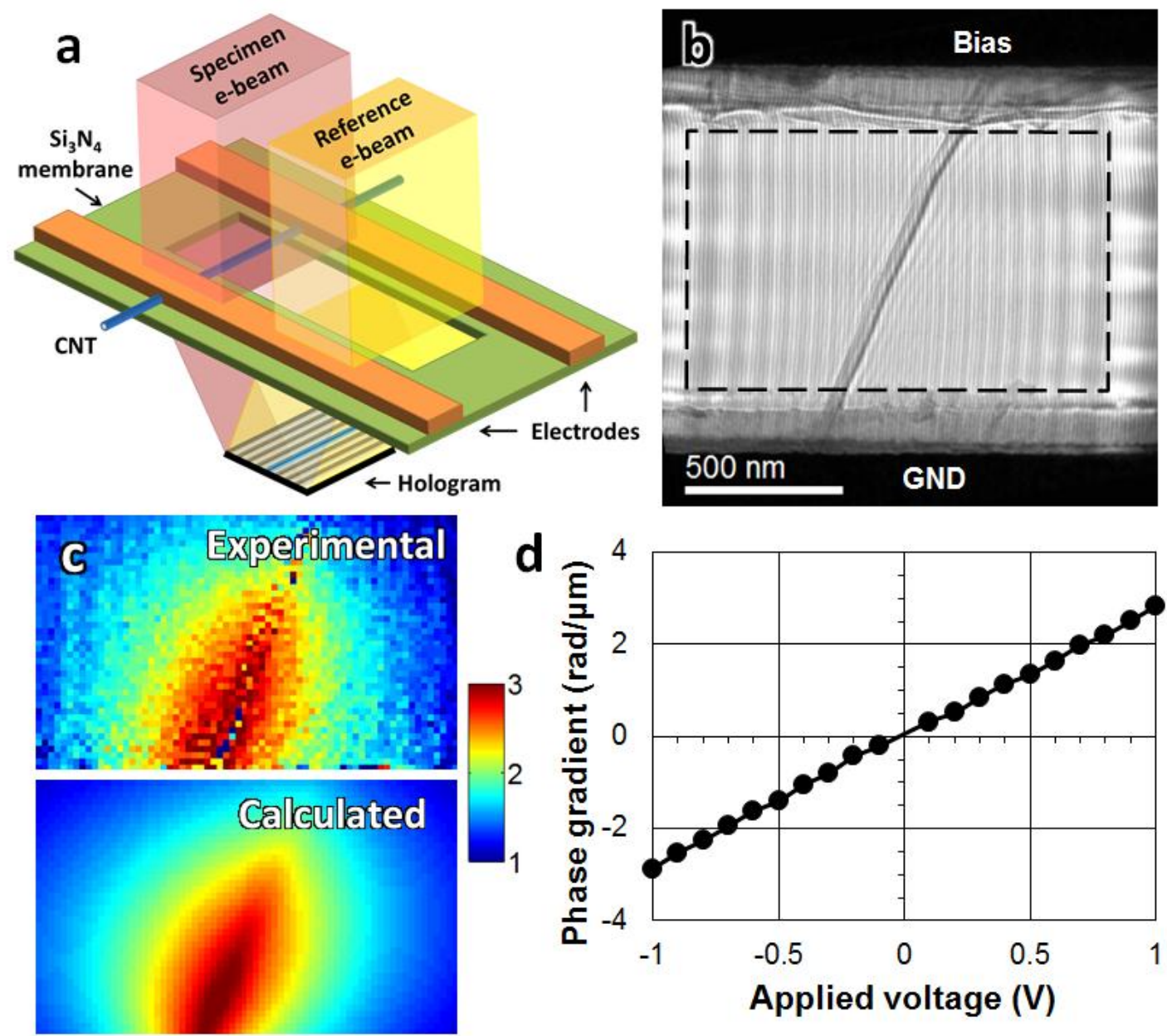

Figure 1. (a) Schematics of in-situ electrical biasing design for EH measurements. A CNT is suspended on an etched slit of a silicon nitride TEM membrane, and electron beams passing through CNT and reference regions experience the same electric fields between the two parallel electrodes. (b) Electron hologram recorded on the CNT in operando mode. (c) Experimental and calculated phase images showing quantitative consistency at external bias of $1 \mathrm{~V}$. (d) Phase gradient as function of applied voltage showing linear dependence. 